\title{
The effect of holes in the dispersion relation of propagative surface plasmon modes of nanoperforated semitransparent metallic films
}

\author{
R. Kekesi, ${ }^{a}$ D. Meneses-Rodríguez, F. García-Pérez, M. U. González, A. García-Martín, \\ A. Cebollada, and G. Armelles ${ }^{\mathrm{b}}$ \\ IMM-Instituto de Microelectrónica de Madrid (CNM-CSIC), Isaac Newton 8, PTM, E-28760 Tres Cantos, \\ Madrid, Spain
}

(Received 11 June 2014; accepted 23 September 2014; published online 2 October 2014)

\begin{abstract}
We have analysed the effect that holes have on the properties of propagative surface plasmon modes in semitransparent nanoperforated Au films. The modes have been excited in Kretschmann configuration. Contrary to continuous films, where only one mode is excited, two modes are observed in Au nanohole array. The origin of this different behavior is discussed using effective optical properties for the nanoperforated films. The presence of the holes affects the effective optical constants of the membranes in two ways: it changes the contribution of the free electrons, and it gives rise to a localized transition due to a hole induced plasmon resonance. This localized transition interacts with the propagative surface plasmon modes, originating the two detected modes. C 2014 AIP Publishing LLC. [http://dx.doi.org/10.1063/1.4897198]
\end{abstract}

\section{INTRODUCTION}

In the last few years, nanoperforated metallic films have attracted much attention from both fundamental and applied points of view. Such structures present new optical phenomena and have found applications in sensing, energy harvesting, and telecom fields. ${ }^{1-7}$ For thick films (typically a few hundreds of nanometers), most of these studies are focused on the effect that different parameters, such as hole diameter, hole shape, periodicity, and degree of ordering of the hole array, etc., have on the optical transmission properties of the structure, with extraordinary transmission effects receiving special attention. ${ }^{8,9}$ However, for partially transparent thin films (typically a few tens of nanometers), where extraordinary transmission phenomena do not apply, ${ }^{10,11}$ the focus was not only on the transmission properties of the system but also on other optical properties such as scattering or reflectance. ${ }^{12-18}$ In all of these studies, it has been clearly shown that propagative plasmon modes, also known as surface plasmon polaritons (SPPs), play a key role in determining the optical properties of the system. However, despite their relevant function, not so much attention has been paid to the modification of these SPP modes induced by the presence of holes, other than the role of corrugated periodicity. In this work, we address this question by analysing the effect that holes have on the dispersion curve of SPP modes of randomly perforated $\mathrm{Au}$ thin films. This is done by exciting SPPs in the so called Kretschmann configuration. ${ }^{19}$ We will show that, contrary to continuous films, where the angular reflectivity curves have only one minimum, the reflectivity curves of the membranes show two minima. These two minima originate from the interaction of the propagative plasmon modes of the membrane and the hole induced plasmon resonance.

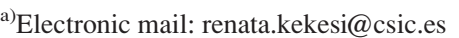

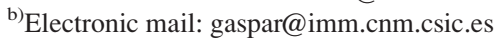

\section{RESULTS}

The membrane samples were obtained using a partial process of the hole mask colloidal lithography technique. ${ }^{20}$ As sketched in Fig. 1, a layer of polystyrene spheres, $200 \mathrm{~nm}$ in diameter, was deposited by spin coating on top of a $200 \mathrm{~nm}$ thick PMMA layer previously deposited on a glass substrate. Then, Au films either 30 or $45 \mathrm{~nm}$ thick were deposited on top by magnetron sputtering, and finally the spheres were removed by tape stripping. The resulting structures consist of a random distribution of $200 \mathrm{~nm}$ diameter nanoholes in a Au layer (in other words, Au membranes) on a continuous PMMA layer deposited on glass. Unless otherwise stated, the hole concentration was around 3 holes $\mu \mathrm{m}^{-2}$. An AFM image of a representative membrane is presented also in Fig. 1 for illustration. Together with the membranes, continuous Au thin films of the same thickness were also deposited as a reference.

In order to study the effect that holes have in the dispersion relation of the SPPs sustained by the Au membranes, we have performed wavelength dependent reflectance measurements in Kretschmann configuration, ${ }^{19}$ combined with spectral ellipsometry. The upper sketch in Fig. 2(a) illustrates schematically the experimental setup. The glass substrate is attached to a semi spherical BK7 prism using matchingindex oil. A p-polarized collimated monochromatic light beam illuminates the sample through the prism and the reflected light is detected with a photodiode. In this configuration, the prism adds the extra momentum that needs the incident photon to couple to the SPP mode propagating along the film/air interface. At the incident angle at which the inplane wavevector of the photon matches the wavevector of the SPP, the reflectivity has a minimum. In Figs. 2(a) and 2(b), we show the reflectivity spectra as a function of the photon energy for the 30 and $45 \mathrm{~nm}$ thick Au membranes, respectively, measured at three different incidence angles. For reference, the corresponding spectra for the respective continuous Au films are also shown. As it can be observed, 


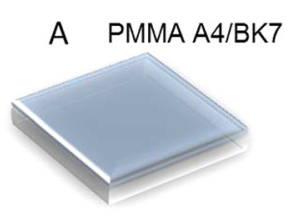

D PS colloidal particles

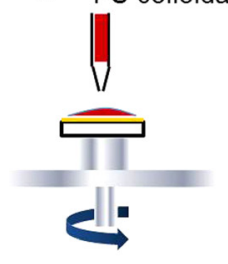

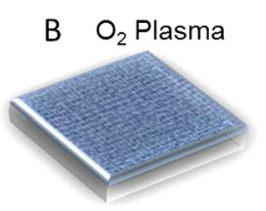

E Au Coat

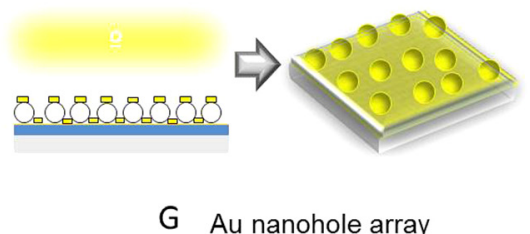

G Au nanohole array
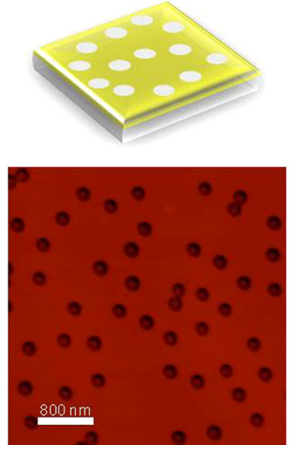

the spectra obtained for the membranes present clear differences with respect to the continuous films. These last ones exhibit a single sharp reflectivity minimum whose position blue-shifts with increasing incidence angles, whereas the reflectivity spectra of the membranes exhibit two somehow broader minima, the high energy one basically obtained at the same energy than that obtained in the continuous film. In the case of the membranes, the spectral positions of both minima blue-shift with increasing incidence angles.

To understand the behaviour obtained for the membranes, we have studied the optical properties of the fabricated structures by spectral ellipsometry. In Fig. 3, we present the effective dielectric constants obtained in this way for both membranes, together with the corresponding constants of a continuous Au layer. As it can be observed, two spectral regions are clearly distinguished: a high energy one, between 2.2 and $5 \mathrm{eV}$, where the optical constants of membranes and continuous films are very similar; and a low energy one, between 0.7 and $2.2 \mathrm{eV}$, where the values of the optical constants of the membranes are different from those of the continuous $\mathrm{Au}$ film. In particular, the absolute value of the real part of the dielectric constant, $\varepsilon_{1}$, is lower for the membranes than for the continuous films, which is an indication of the lower metallic character of the membranes. The value of the imaginary part, $\varepsilon_{2}$, on the contrary, is higher for the membranes. Moreover, a feature around $1.5 \mathrm{eV}$, the same spectral region where the additional broad minimum was observed in the reflectance versus photon energy measurements shown in Fig. 2, is clearly observed for the membranes. This feature appears as a peak in the imaginary part of the membranes dielectric constants, and as an s-like contribution in the real part. In both real and imaginary parts, the feature position appears red-shifted in the $30 \mathrm{~nm}$ membrane with respect to the $45 \mathrm{~nm}$ one. It also red-shifts with
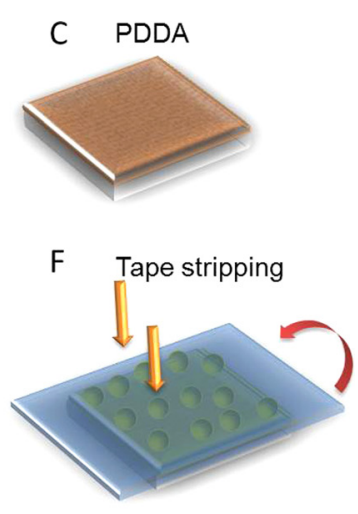

FIG. 1. Sketch of the Au membrane fabrication process, and AFM image of the final structure obtained. the decrease of the hole concentration, as it can be seen in Fig. 4, where we present the effective optical constants of $45 \mathrm{~nm}$ thick membranes for two holes concentrations of 3 and 0.7 holes $\mu \mathrm{m}^{-2}$ (blue and red solid lines, respectively). Therefore, both the energy range where this feature appears as well as its dependence with the membrane thickness and hole concentration strongly suggest that this structure is related to a nanohole induced plasmon resonance similar to the one observed in Refs. 12-18 by other groups.

From the values of the effective dielectric constants in the spectral range $0.7-2.2 \mathrm{eV}$ for different holes concentration shown in Fig. 4, we can see that the absolute value of the real part increases as we decrease the hole concentration, indicating a more metallic character. The value of the imaginary part, on the other hand, decreases. This behavior can be easily understood if we consider that in this spectral range the main contribution to the optical properties of continuous $\mathrm{Au}$ films comes from the conduction electrons, which can be described using the Drude model as ${ }^{21}$

$$
\varepsilon(\omega)=\varepsilon_{\infty}-\frac{\omega_{p}^{2}}{\omega^{2}+i \Gamma \omega},
$$

where $\omega_{\mathrm{p}}$ is the plasma frequency $\left(\omega_{p}^{2}=\frac{n e^{2}}{m^{*} \varepsilon_{0}}\right.$, being $\mathrm{n}$ the electron concentration, and $\mathrm{m}^{*}$ the effective mass), $\Gamma$ the relaxation frequency of the electrons, and $\varepsilon_{\infty}$ takes into account the interband transitions located at higher energy. The black dotted lines shown in Fig. 4 correspond to the dielectric constants obtained using Eq. (1) with $\mathrm{w}_{\mathrm{p}}=8.6 \mathrm{eV}$, $\Gamma=0.7 \mathrm{eV}$, and $\varepsilon_{\infty}=7.3$, which reproduces very well the experimental results for a continuous film represented by the solid black curves. The contribution of the conduction electrons for the membranes can also be modelled using this approach. However, to take into account the contribution of 

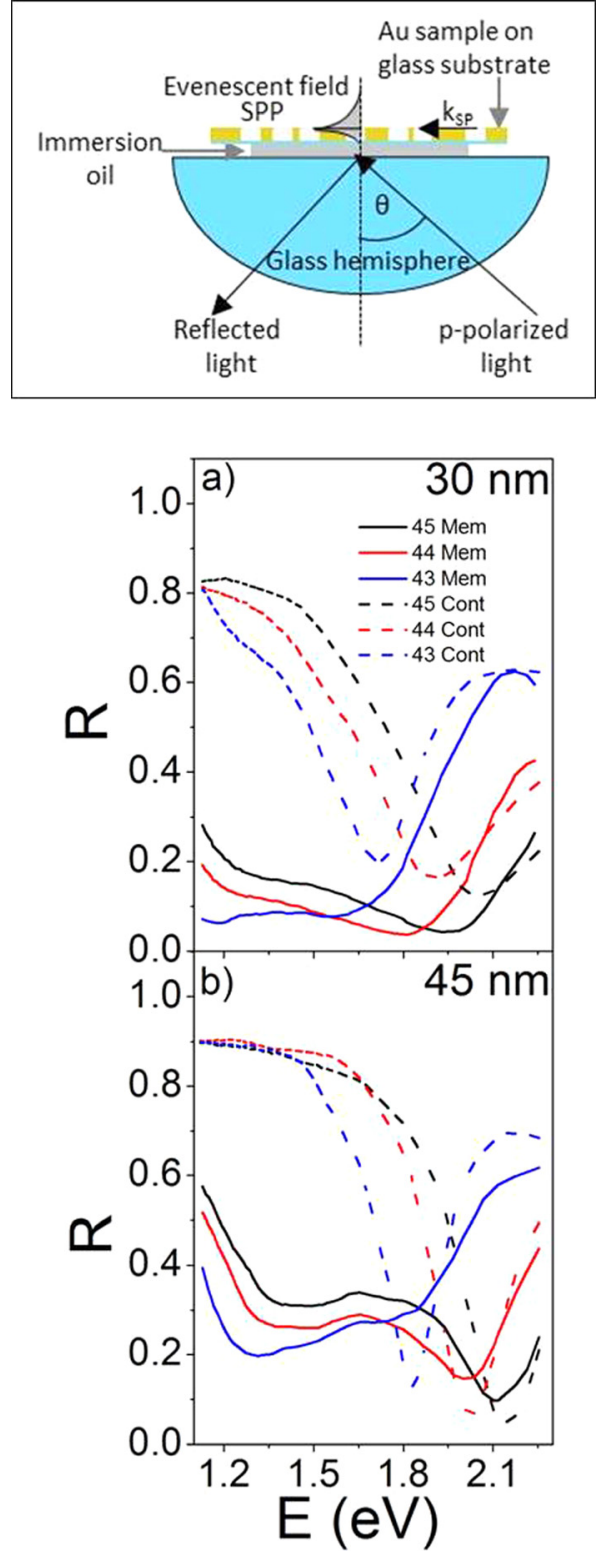

FIG. 2. Top sketch of the Kretschmann configuration measurement setup. Reflectance versus photon energy curves measured in Kretschmann configuration at incidence angles of $43^{\circ}$ (black), $44^{\circ}$ (red), and $45^{\circ}$ (blue) for (a) $30 \mathrm{~nm}$ and (b) $45 \mathrm{~nm}$ thick layer structures. Solid lines correspond to membrane structures and dashed lines to continuous film of identical thickness.

the hole-plasmon resonance localized transition, a Gaussian functional form, $\mathrm{f}(\mathrm{w})$, must be introduced. So, the blue and red dotted curves of Fig. 4 correspond to fits to the experimental dielectric constants of the membranes with different hole concentration (solid blue and red curves) using these two Drude and localized transition contributions

$$
\varepsilon(\omega)=\varepsilon_{\infty}-\frac{\omega_{p}^{2}}{\omega^{2}+i \Gamma \omega}+\mathrm{f}(\omega) .
$$

As it can be observed, the frequency dependence of the dielectric constants is well described by Eq. (2). The inset to Fig. 4(a) depicts the dependence of $\mathrm{w}_{\mathrm{p}}{ }^{2}$ and $\Gamma$ on the holes concentration. As the hole concentration increases, $\mathrm{w}_{\mathrm{p}}{ }^{2}$ decreases and $\Gamma$ increases. The decrease of $\mathrm{w}_{\mathrm{p}}{ }^{2}$ is related to a reduction of the electron concentration, whereas the raise

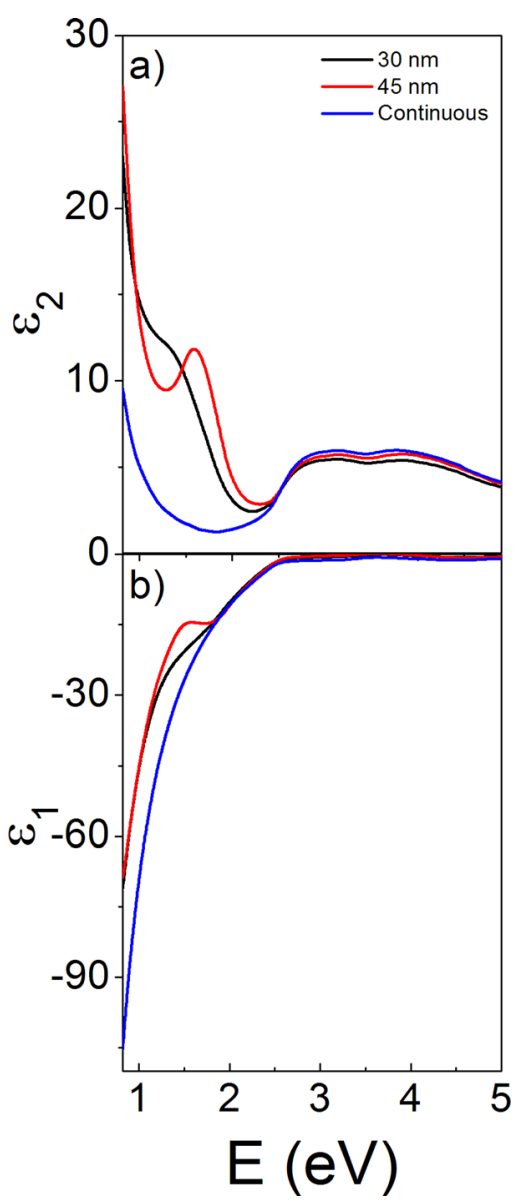

FIG. 3. Imaginary (a) and real parts (b) of the effective dielectric constants determined from ellipsometry measurements for a $30 \mathrm{~nm}$ thick membrane (black), $45 \mathrm{~nm}$ thick membrane (red), and continuous Au film (blue).

of $\Gamma$ is related to the decrease of the scattering time of the electrons with the number of holes. Indeed, the decrease of the electron concentration, $\mathrm{n}$, as well as the increase in the number of scattering centers are expected to be proportional to the number of holes. This suggests, as a first approximation, linear dependencies of $\mathrm{w}_{\mathrm{p}}^{2}$ and $\Gamma$ with the holes density, as it can be seen in the graph. Finally, $\varepsilon_{\infty}$ is similar to that of the continuous film for all the membranes, since its origin is in higher frequency interband transitions, which are not modified by the presence of holes. Regarding the localized transition, $\mathrm{f}(\mathrm{w})$, which takes into account the nanohole induced plasmon resonance, its intensity decreases and redshifts as we decrease the hole concentration.

Therefore, from the point of view of the effective dielectric constants, the effect of the holes on the optical properties of continuous Au films is twofold: on the one hand, they modify the contribution of the free electrons to the optical properties by changing the plasma frequency and the scattering time, and on the other hand they introduce an additional localized transition due to a hole induced plasmon resonance.

With the effective dielectric constants obtained by ellipsometry, it is possible now to calculate the spectral dependence of the reflectance of the different membranes and continuous films as measured in Kretschmann configuration and compare with the experimental results presented in Fig. 2. The obtained results are shown in Fig. 5. Figure 5(a) 


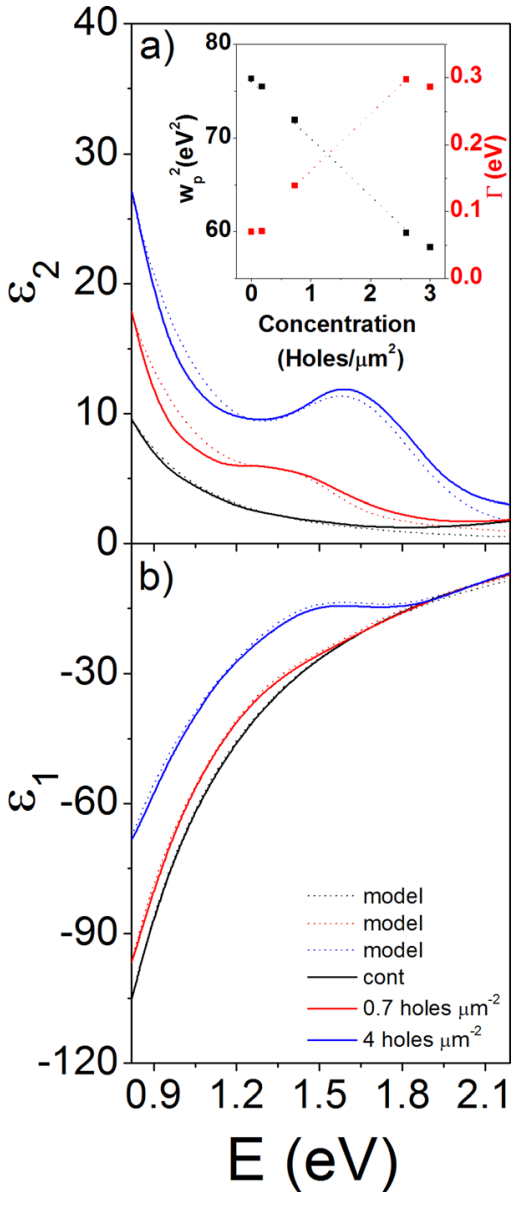

FIG. 4. Imaginary (a) and real parts (b) of the effective dielectric constants determined from ellipsometry measurements for a $45 \mathrm{~nm}$ thick membrane for different hole concentrations. Blue: 3 holes $/ \mu \mathrm{m}^{2}$; red: 0.7 holes $\mu \mathrm{m}^{-2}$; black: $45 \mathrm{~nm}$ continuous film. Solid lines correspond to values determined from ellipsometry measurements, while dashed lines correspond to fits including a Drude term and a localized transition. The inset on the upper panel shows the evolution of the relaxation frequency, $\Gamma$, (red) and the plasma frequency, $\omega_{\mathrm{p}}{ }^{2}$, (black) as a function of the hole concentration. The red and black dotted lines are shown as a guide for the eyes.

shows a reflectivity map between $35^{\circ}$ and $50^{\circ}$ incidence angles for the $45 \mathrm{~nm}$ thick membrane, while Figs. 5(b) and 5 (c) present the reflectivity curves for 30 and $45 \mathrm{~nm}$ thick structures for the same incidence angles used in the experiments. As it can be seen, the agreement of the curves with the experimental results presented in Fig. 2 is excellent, with the two broad minima well reproduced. The reflectivity map offers a clear insight of the meaning of these minima. The portion of the map below the critical angle (incidence angles smaller than $41^{\circ}$ ), where SPP excitation is not possible, shows a non-dispersive minimum around $1.5 \mathrm{eV}$. This minimum can then be associated with the localized transition due to the holes. Above the critical angle, on the other hand, the SPP can be excited and it can be observed an interaction between the localized transition and the SPP mode, ${ }^{22-24}$ which gives rise to the two dispersive modes that have been detected in the reflectivity curves.

Finally, in Figure 6(a) we present the SPP dispersion curves for two $45 \mathrm{~nm}$ thick films. This dispersion curve was calculated considering a system where a metallic layer was embedded between two dielectric layers. ${ }^{25}$ The dielectric
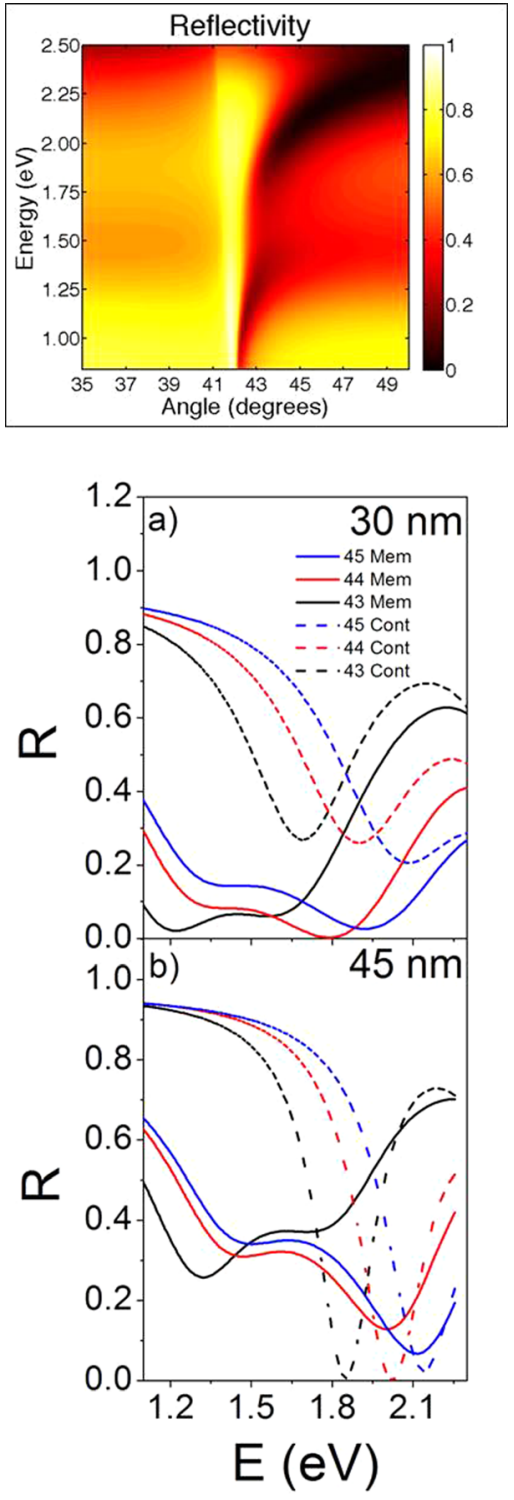

FIG. 5. (a) Reflectance map in Kretschmann configuration for the $45 \mathrm{~nm}$ thick membrane, plotted between $35^{\circ}$ and $50^{\circ}$ incidence angles. Reflectance vs. photon energy curves calculated for 30 (a) and $45 \mathrm{~nm}$ (b) thick membranes under identical conditions than experimental results shown in Fig. 2. The effective dielectric constants determined from ellipsometry measurements are used as input parameters for the calculations.

constant of the metallic layer was determined from the fitting of the ellipsometry measurement for the membrane (red line) and gold continuous layer (black line), respectively. The SPP dispersion curve of the layer with the optical constants of the Au continuous film shows a monotonic increase of the frequency with the wavevector, whereas the SPP dispersion curve of the layer with the optical constants of the membrane has now a s-like structure due to the hole-plasmon feature (see inset of Figure 6(a) where we present the difference between the two curves). Moreover, from the effective dielectric constants the vertical decay of the SPP electromagnetic field intensity in both air and metal can be also calculated. Figure 6(b) shows such decay constants for both membrane and continuous $\mathrm{Au}$ film as a function of the frequency. As it can be seen, in the spectral region where the optical properties are modified by the presence of holes, the 

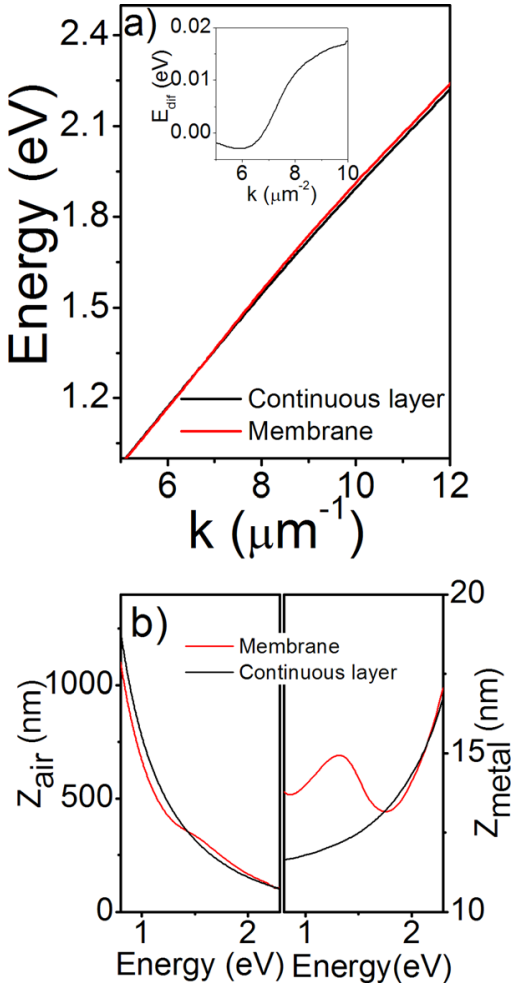

FIG. 6. (a) SPPs dispersion relation calculated for a continuous 45 thick film (black line) and a $45 \mathrm{~nm}$ thick membrane (red line), using the effective dielectric constants determined by ellipsometry. The inset corresponds to the difference between the two curves. (b) Vertical decay constants of the SPP intensity in air and in metal as a function of the energy for both a membrane and a continuous Au film.

localization of the SPP modes changes: the extension of the electromagnetic (EM) field in the air decreases, whereas the extension in the metal increases. This behaviour corroborates the interpretation of the two minima in the reflectance as due to the interaction of the propagative plasmon modes of the membrane and the hole induced plasmon mode. ${ }^{22-24}$ Due to this interaction, both modes hybridize and as a consequence their degree of localization changes. The EM field of the hole plasmon mode is localized in the hole and, therefore, the resulting modes are more localized in the equivalent metal layer than those of the continuous film. Similar phenomenology has been recently found in quasicontinuous metallic monolayers by Yun et al., ${ }^{26}$ where a progressive change in the reflectivity spectra has been observed when going from continuous metallic layers to isolated metallic nanoparticles. In this work, also two minima in the reflectivity were observed in the intermediate case where the nanoparticles are strongly interacting, and they were interpreted in terms of the hybridization of propagative plasmons and localized plasmons due to the metallic nanoparticles. In our case, the contribution of the metallic particles is substituted by the insertion of holes in the metallic layer.

\section{SUMMARY AND CONCLUSIONS}

In conclusion, we have analysed the effect that holes have on the propagative surface plasmon modes of semitransparent nanoperforated Au films. We have shown that holes modify the effective dielectric constant of Au films by changing the free electron contribution and introducing a new localized plasmon resonance. In the spectral region of this resonance, the dispersion curve of the propagative surface plasmon modes of the membranes is modified due to interaction with this localized mode, and it becomes less dispersive.

\section{ACKNOWLEDGMENTS}

Funding from Spanish Ministry of Economy and Competitiveness through grants "FUNCOAT" CONSOLIDER CSD2008-00023, MAPS MAT2011-29194C02-01, and from Comunidad de Madrid through grant “MICROSERES-CM" S2009/TIC-1476 is acknowledged.

${ }^{1}$ M. P. Jonsson, A. B. Dahlin, P. Jönsson, and F. Höök, Biointerphases 3, FD30 (2008).

${ }^{2}$ R. Gordon, D. Sinton, K. L. Kavanagh, and A. G. Brolo, Acc. Chem. Res. 41, 1049 (2008).

${ }^{3}$ J. F. Masson, M. P. Murray-Method, and L. S. Live, Analyst 135, 1483 (2010).

${ }^{4}$ T. H. Reilly, R. C. Tenent, T. M. Barnes, K. L. Rowlen, and J. van de Lagemaat, ACS Nano 4, 615 (2010).

${ }^{5}$ J. L. Skinner, A. A. Talin, and D. A. Horsley, J. Vac. Sci. Technol., B 26, 2139 (2008).

${ }^{6}$ J. L. Skinner, A. A. Talin, and D. A. Horsley, Opt. Express 16, 3701 (2008).

${ }^{7}$ T. H. Reilly, J. van de Lagemaat, R. C. Tenent, A. J. Morfa, and K. L. Rowlen, Appl. Phys. Lett. 92, 243304 (2008).

${ }^{8}$ F. J. García de Abajo, Rev. Mod. Phys. 79, 1267 (2007).

${ }^{9}$ F. J. Garcia-Vidal, L. Martin-Moreno, T. W. Ebbesen, and L. Kuipers, Rev. Mod. Phys. 82, 729 (2010)

${ }^{10}$ C. Genet and T. W. Ebbesen, Nature 445, 39 (2007)

${ }^{11}$ R. Gordon, A. G. Brolo, D. Sinton, and K. L. Kavanagh, Laser Photon. Rev. 4, 311 (2009).

${ }^{12}$ J. Prikulis, P. Hanarp, L. Olofsson, D. Sutherland, and M. Käll, Nano Lett. 4, 1003 (2004).

${ }^{13}$ T. Rindzevicius, Y. Alaverdyan, B. Sepulveda, T. Pakizeh, M. Käll, R. Hillenbrand, J. Aizpurua, and F. J. García de Abajo, J. Phys. Chem. C 111, 1207 (2007).

${ }^{14}$ Y. Alaverdyan, B. Sepúlveda, L. Eurenius, E. Olsson, and M. Käll, Nat. Phys. 3, 884 (2007).

${ }^{15}$ B. Sepúlveda, Y. Alaverdyan, J. Alegret, M. Käll, and P. Johansson, Opt. Express 16, 5609 (2008).

${ }^{16}$ T. H. Park, N. Mirin, J. B. Lassiter, C. L. Nehl, N. J. Halas, and P. Nordlander, ACS Nano 2, 25 (2008).

${ }^{17}$ T. Sannomiya, O. Scholder, K. Jefimovs, C. Hafner, and A. B. Dahlin, Small 7, 1601 (2011).

${ }^{18}$ M. Schwind, V. D. Miljković, M. Zäch, M. Käll, I. Zorić, and P. Johansson, ACS Nano 6, 9455 (2012).

${ }^{19}$ E. Kretschmann and H. Raether, Z. Naturforsch., A 23A, 2135 (1968).

${ }^{20}$ H. Fredriksson, Y. Alaverdyan, A. Dmitriev, C. Langhammer, D. Sutherland, M. Zäch, and B. Kasemo, Adv. Mater. 19, 4297 (2007).

${ }^{21}$ M. A. Ordal, R. J. Bell, R. W. Alexander, L. L. Long, and M. R. Querry, Appl. Opt. 24, 4493 (1985).

${ }^{22}$ J. F. Torrado, J. B. Gonzalez-Diaz, M. U. Gonzalez, A. Garcia-Martin, and G. Armelles, Opt. Express 18, 15635 (2010).

${ }^{23}$ A. Christ, T. Zentgraf, S. G. Tikhodeev, N. A. Gippius, J. Kuhl, and H. Giessen, Phys. Rev. B 74, 155435 (2006).

${ }^{24}$ Y. Chu and K. B. Crozier, Opt. Lett. 34, 244 (2009).

${ }^{25}$ S. A. Maier, Plasmonics: Fundamentals and Applications (Springer, 2007), p. 30

${ }^{26}$ H. Yun, M. II-Lee, S. Y. Lee, K. Y. Kim, and B. Lee, Sci. Rep. 4, 3696 (2014). 